\title{
Linx
}

Revue des linguistes de l'université Paris X Nanterre

$80 \mid 2020$

L'héritage de Jean Dubois et Françoise Dubois-Charlier

\section{Les manuels de Jean Dubois}

Jean Dubois' textbooks

\section{Gaston Gross}

\section{OpenEdition}

Journals

Édition électronique

URL : http://journals.openedition.org/linx/5388

ISSN : 2118-9692

Éditeur

Presses universitaires de Paris Nanterre

Référence électronique

Gaston Gross, « Les manuels de Jean Dubois », Linx [En ligne], 80 | 2020, mis en ligne le 10 juillet 2020, consulté le 05 août 2020. URL : http://journals.openedition.org/linx/5388 ; DOI : https://doi.org/ $10.4000 / \operatorname{linx} .5388$

Ce document a été généré automatiquement le 5 août 2020

Département de Sciences du langage, Université Paris Ouest 


\title{
Les manuels de Jean Dubois
}

\author{
Jean Dubois' textbooks
}

\section{Gaston Gross}

1 On a de la peine à imaginer, de nos jours, ce que représentait dans les années 60 la vague constituée par l'introduction de la linguistique scientifique dans un grand nombre de pays. Pour ne parler que de la France, on peut citer les travaux de B. Quemada à Besançon sur l'informatisation du lexique et son rôle central dans le traitement automatique. En 1958, il fonde la revue Les Cahiers de Lexicologie, qui a contribué à l'essor de cette discipline. On peut ajouter la création du Trésor de la langue française, par Paul Imbs, dirigé ensuite par B. Quemada puis par Robert Martin. On doit adjoindre les travaux de Charles Muller sur les statistiques linguistiques et la mise au jour d'outils efficaces de description du lexique. On doit évoquer aussi les recherches de Maurice Gross dans le cadre du LADL et son rôle dans le traitement automatique, en particulier du lexique et plus particulièrement du figement et son rôle dans l'introduction en France des travaux de Z. Harris. Je voudrais ajouter à cette liste les travaux de Jean Dubois, qui entre 1965 et 1970, a publié quatre livres qui ont eu une importance particulière dans l'élaboration de la linguistique en France.

\section{Grammaire structurale du français : nom et pronom (1965)}

2 Ce premier ouvrage (190 p.) porte sur les groupes nominaux, et plus particulièrement sur le nombre, le genre, les articles et les substituts (pronoms). L'introduction se réclame explicitement de l'analyse structurale du français contemporain et du "fonctionnement d'un système codique spécifique». Une réflexion de nature sociologique définit une norme, source de la description, qui présente un français « neutralisé ", écartant les cas marqués. Vient ensuite une présentation de la méthode distributionnelle. Jean Dubois cite Z. Harris et L. Bloomfield et mentionne l'attitude béhavioriste que ce dernier avait introduite dans les études de langue: «l'objet 
premier de l'analyse distributionnelle est descriptiviste ; elle vise à offrir une description totale d'un état de langue en synchronie ».

3 Cet objectif repose sur certains principes: le caractère achevé du corpus, considéré comme représentatif de l'ensemble de la langue; et le fait que le sens d'un message ne peut être défini indépendamment d'une situation de communication donnée. La description linguistique, fondée sur l'analyse distributionnelle, ne peut donc pas prendre le sens pour base de l'analyse. Enfin, la méthode repose sur l'analyse syntagmatique des segments, c'est-à-dire leur position sans la chaîne parlée. L'analyse distributionnelle a permis la détermination des phonèmes. Une rapide allusion à $\mathrm{N}$. Chomsky n'éloigne pas la description d'une stricte analyse distributionnelle que J. Dubois associe à la théorie de la communication avec les notions de redondance et de bruit, définies grâce aux termes de récepteur et émetteur ou encore d'encodage et de décodage. Une analyse linguistique repose sur une distribution des marques dans l'énoncé.

4 Sont ensuite mis en évidence les rangs de l'analyse linguistique sur la base de la notion de la double articulation d'A. Martinet : celle des unités significatives minimales et celle des phonèmes. Vient ensuite la définition des concepts de base de l'analyse linguistique : syntagme, phrase, énoncé, phrase minimale, syntagme nominal, syntagme verbal ainsi que la notion de constituants immédiats. Comme on le voit, on est dans le cadre d'une grammaire distributionnelle classique. Le livre lui-même, comme l'indique le sous-titre, comprend trois parties portant sur le nombre (p. 17 à 50), le genre (p. 51 à 90), les substituts (p. 91 à 190).

5 La première partie du livre est donc consacrée au nombre dans les groupes nominaux du français (singulier/pluriel) à la fois dans le code écrit et dans le code oral, ainsi qu'à la distribution des marques dans la phrase. Sont étudiés ensuite différents facteurs : la redondance des marques à l'écrit et à l'oral ainsi que les expansions.

6 La seconde partie du livre est consacrée au genre. J. Dubois étudie les différentes fonctions de l'opposition jouées par le genre dans l'organisation de la langue : indication du sexe pour les animés et, dans certains cas, pour les inanimés. L'opposition de genre est étudiée dans le détail du point de vue de la morphologie des marques. Cette partie est particulièrement claire et méticuleuse. Une remarque à la fin de ce chapitre mérite d'être signalée : «Il y a interdépendance entre les phénomènes du lexique et ceux de la grammaire ».

7 La troisième partie est consacrée aux substituts, qui correspondent à une réduction du coût du message. Après une définition du phénomène, sont étudiées les différentes classes de substituts remplaçant un groupe nominal (pronom) ou un syntagme verbal (comme le verbe faire). Après une étude des caractéristiques morphologiques des substituts, portant sur les fonctions syntagmatiques des substituts dans le cadre d'une phrase, sont ainsi examinés les pronoms personnels et leurs variantes combinatoires, les possessifs, les articles, les démonstratifs ainsi que les relatifs et les interrogatifs.

Ce premier livre suggère les remarques suivantes. Comme l'indique clairement l'introduction et comme le met en évidence la bibliographie, cet ouvrage se réclame de la grammaire distributionnelle, dont il analyse à la fois les méthodes et les réalisations dans le cadre du groupe nominal. Sont scrutées méticuleusement les réalisations des deux codes (écrit et oral). Il s'agit donc d'une description précise et essentiellement morphologique des divers composants des groupes nominaux. Apparaissent cependant 
çà et là des ouvertures sur d'éventuelles modifications théoriques, comme l'indication dans la bibliographie du livre de N. Chomsky Syntactic Structures.

\section{Grammaire structurale du français : le verbe (1967)}

Deux ans plus tard, en 1967, paraît un second volume : Grammaire structurale du français : le verbe. On peut considérer cet ouvrage comme une étape intermédiaire. Son titre le rattache au volume précédent, dans son interprétation structurale. Cependant, la tonalité de l'introduction est assez différente : «Si la description morphologique relève encore de la méthodologie exposée précédemment, le fonctionnement du verbe a été lui-même analysé dans la perspective d'une grammaire transformationnelle, qui pour nous se situera au niveau des performances réalisées ». A quoi, l'auteur ajoute : «La linguistique, dans ses aspects les plus récents part en effet d'une hypothèse sur le fonctionnement du langage qui suppose une révision complète des modèles jusqu'ici utilisés dans la description d'une langue. Estimant insuffisantes les analyses distributionnelles et les taxinomies qui, appuyées en définitive sur des modèles probabilistes, avaient permis de lui donner un premier statut scientifique, la linguistique s'efforce de rendre compte de la créativité du langage au-delà de l'étude d'un texte fini. La description d'une langue n'est plus l'exposé cohérent de règles de combinaison ordonnées selon des niveaux arbitraires (phonématique, morphématique, phrastique), induites d'un corpus limité et appelées à justifier l'ensemble des énoncés existants. La langue se présente dans cette nouvelle perspective comme un ensemble non fini ».

10 Interviennent alors trois nouvelles notions, qui étaient étrangères aux grammaires distributionnelles. Celle qui permet de passer de deux phrases à une seule par un syntagme nominal, par l'effacement ou l'adjonction d'un segment, par la relativisation, etc. : l'enfant court > la course de l'enfant; le groupe est hétérogène > l'hétérogénéité du groupe; le tapis est rouge > le tapis rouge. Cette notion de transformation implique la présence de "phrases minimales", sources des transformations effectuées par le locuteur. Les transformations ont un degré d'acceptabilité variable. On constate alors "l'existence de classes de termes dont les compatibilités et les incompatibilités sont traduites en termes de corrélations sémantiques et/ou syntaxiques : parmi ces dernières la corrélation animé/non-animé est fondamentale ». La linguistique acquiert dès lors « le statut de science expérimentale ».

11 L'ouvrage est un peu plus conséquent que le précédent (218 pages). Il comprend neuf chapitres :
1. Le verbe et le syntagme verbal
2. La phrase minimale
3. Bases verbales et bases nominales
4. Structure morphologique du verbe. Les conjugaisons du français
5. Transformation passive
6. Transformation négative
7. Hiérarchie des marques dans le verbe et le substantif
8. Adjectif et adjectif adverbial dans le syntagme verbal
9. Adverbes et locutions de temps dans l'énoncé

Ces titres suscitent un certain nombre d'observations. J. Dubois, dans son introduction, se réclame explicitement d'une inspiration générative. On pouvait imaginer que son premier chapitre soit consacré, comme c'est le cas de tous les manuels de cette 
inspiration, à la notion de phrase minimale, comme point de départ de toute analyse. Comme on le voit, il consacre le premier chapitre à la définition du verbe et du syntagme verbal. Une telle hésitation théorique mérite d'être signalée: "Les morphèmes qui appartiennent à la classe des verbes se définissent, relativement aux autres classes, par leurs distributions dans l'énoncé élémentaire et par leurs systèmes spécifiques de marques». On voit que, malgré ce qui vient d'être signalé dans l'introduction, on se situe encore dans un cadre distributionnaliste. Le chapitre 1 définit donc la classe des verbes, la notion de syntagme verbal, de conjugaison, de formes non actualisées (participe et infinitif).

13 Le chapitre 2 est une définition de la phrase minimale, ce qui constitue néanmoins une nouveauté dans ces préoccupations théoriques. Ce chapitre aurait pu commencer le livre, d'autant plus que sa première phrase signale que «l'analyse des distributions spécifiques du verbe implique la référence à l'unité linguistique de rang supérieur, c'est-à-dire la phrase ». Le chapitre sépare les cas où la phrase est une phrase active « achevée » ou " non achevée ». Vient ensuite une suite de critères qui définissent une phrase active achevée : une phrase est définie par un syntagme nominal et un syntagme verbal, qui constituent les constituants immédiats. Le syntagme nominal est le sujet et le syntagme verbal le prédicat. Sont définis ensuite les syntagmes nominaux et les syntagmes verbaux ainsi que les notions de sujet et d'objet. A quoi il faut ajouter les différents types de verbes : verbes actifs, verbes passifs et pronominaux. Le schéma de phrase standard sera donc $\mathrm{P}=\mathrm{SN} 1+\mathrm{V}+\mathrm{SN} 2$. Cette présentation implique deux types de groupes nominaux différents selon leur fonction : un groupe nominal sujet et un groupe nominal objet. Un groupe nominal peut être remplacé par un substitut (pronom). A côté d'une phrase active "achevée», on observe des phrases "nonachevées » auxquelles il manque un élément, essentiellement un argument : il mange des pommes de terre > il mange; il fume une cigarette > il fume. Différentes observations examinent le cas où un argument, essentiellement un complément, peut être effacé.

Le chapitre 3 est consacré aux bases verbales et bases nominales. Il s'agit essentiellement de l'étude des relations existant entre un verbe et les différentes formes nominales qu'il peut avoir, en fonction de ses emplois. Une même racine prédicative peut être exprimée à l'aide d'un verbe, d'un nom prédicatif ou encore par un adjectif. D'autre part, l'effacement d'un verbe support permet la transformation d'une phrase minimale en en syntagme nominal, relation dont J. Dubois établit les différentes règles. Vient ensuite une description des règles morphologiques reliant les bases nominales et les bases verbales. Le caractère minutieux de ce travail de description morphologique mérite d'être signalé.

Le chapitre 4 est consacré à la structure morphologique du verbe, c'est-à-dire aux différentes conjugaisons du français. Après une rapide classification traditionnelle (et critique) de la flexion des verbes correspondant à trois types de conjugaisons, où l'on oppose les paradigmes réguliers (-er, -ir) aux paradigmes irréguliers (-ir, -re, -oir), les critères retenus dans l'analyse des verbes sont les suivants $: a)$ distinction entre langue parlée et langue écrite ; b) distinction entre formes simples et formes composées c) variation des bases verbales (radicaux) comme principe essentiel du classement. Ces descriptions sont mises en pratique dans la description de la " première » conjugaison, celle du verbe être (p. 60-63). Les deuxième et troisième conjugaisons correspondent à ceux des verbes qui ont, dans leur paradigme, 6 ou 5 radicaux. Il s'agit de verbes usuels (aller, prendre, avoir, pouvoir, vouloir, etc.). La quatrième conjugaison, de classe limitée, 
correspond à des verbes à 4 bases : savoir, venir, tenir, prendre, valoir, comprendre. Là aussi, les formes des différents temps sont étudiées dans le détail. La cinquième conjugaison comprend des verbes à 3 bases (radicaux) : devoir, recevoir, boire, connaître, paraitre, croître, etc. La sixième classe est caractérisée par des verbes à deux bases. Elle comprend trois sous-classes qui sont décrites dans le détail, en fonction des oppositions de formes. Enfin, la septième conjugaison concerne les verbes qui sont construits sur un seul radical, souvent issus de radicaux nominaux. Cette classe correspond (approximativement) à la première conjugaison traditionnelle. Ce chapitre constitue donc une étude des paradigmes des différentes conjugaisons.

Le chapitre 5 est consacré à la transformation passive. Comme on le voit au titre même, la notion de transformation implique que l'auteur a changé, en partie du moins, sa position théorique puisque la notion de transformation ne figurait pas explicitement dans le premier volume. Le chapitre est assez conséquent (pp. 80 à 131). Il commence par une définition : «On appelle transformation passive de l'énoncé minimal l'application à ce dernier d'une règle de transformation passive telle que, le signifié étant invariant, le rôle respectif et l'ordre des deux syntagmes nominaux soient inversés ». Sont ensuite étudiées les limitations de la transformation passive (qui est plus coûteuse que la forme active correspondante). (p. 104).

Le chapitre 6 traite de la transformation négative et des différentes formes qu'elle peut recouvrir dans une phrase donnée. Après une définition de la transformation négative, l'auteur examine successivement les moyens morphologiques de la négation (à l'oral et à l'écrit) ainsi que son expression avec les différentes catégories prédicatives (noms, verbes, adjectifs), et avec d'autres modifications, comme l'emphase.

Le chapitre 7 est intitulé : «Hiérarchie des marques dans le verbe et le substantif ». Il est consacré essentiellement au temps et à l'aspect, en particulier dans le cadre de l'opposition entre énoncé et récit. Les considérations morphologiques (marques verbales) y sont étudiées dans le détail. Les deux derniers chapitres, plus courts, sont consacrés à l'adjectif et à ses propriétés (Chap. 8) et aux adverbes (Chap. 9).

\section{Grammaire structurale du français : la phrase et les transformations (1969)}

19 Ce troisième volume constitue en fait le changement déterminant dans les positions théoriques de l'auteur. S'il s'intitule encore " grammaire structurale du français », c'est par référence aux deux précédents, qui portaient ce titre. En effet, l'introduction s'ouvre par une mise au point théorique : «Ce troisième volume de la Grammaire du français aborde les problèmes de syntaxe dans une perspective différente de la méthode suivie dans les deux premiers tomes. C'est ce que souligne l'apparition dans le titre du terme transformation à côté de l'adjectif structural, puisque celui-ci ne répondait plus aux principes de l'analyse mise en œuvre pour rendre compte des transformations de la phrase de base, connues sous le nom de dérivation. Aussi est-il nécessaire de tracer les grandes lignes qui séparent les méthodes distributionnelles et structurales de celles qui servent de base aux études actuelles de grammaire transformationnelles ».

Cet ouvrage est précédé d'une introduction théorique de 15 pages, dont l'objectif est de faire le point sur l'état de la théorie en 1969. Comme il s'agit d'un changement de théorie de la part de l'auteur, cette introduction résume les acquis de la linguistique 
dite "structurale» et une réflexion sur les acquis et les failles de cette position théorique. Ces quelques pages sont en fait un tableau panoramique d'une grande clarté des travaux de linguistique de la première moitié du $20^{\mathrm{e}}$ siècle. J. Dubois définit avec une grande clarté les principes de la grammaire structurale, telle qu'elle a été pratiquée en Europe au siècle dernier et dont il va se démarquer dans ce livre. Ce texte est particulièrement dense. La théorie structurale s'applique à des énoncés réalisés. C'est à la fois une théorie du texte comme objet clos et une méthode d'analyse formelle qui doit rendre compte de la structure d'un énoncé. Le linguiste limite volontairement son objet à l'étude des énoncés réalisés, dont il définit la structure. Sont exclus le sujet et la situation, qui sont rejetés du domaine de la linguistique.

21 A partir de ces considérations générales, J. Dubois examine les écrits des auteurs les plus marquants de la théorie structurale tant en Europe qu'en Amérique : L. Bloomfield, Y. Bar-Hillel, R. Jakobson, E. Benveniste, F. de Saussure, A. Bally ; U. Weinrich ; L. Hjelmslev et V. Brøndal. Vient ensuite un résumé conclusif de la théorie structurale qui met l'accent sur le principe d'immanence du texte, qui distingue langue et parole. Il s'agit de définir la combinatoire décrite par les règles. La description structurale est synchronique. Le signe linguistique est arbitraire.

La méthode structurale se fonde sur une théorie des niveaux. La langue se présente comme une série de rangs hiérarchisés (phonologique, morphologique, phrastique) où chaque unité est définie par ses combinaisons dans le rang supérieur. Les descriptions se fondent donc sur les notions de phonèmes, de morphèmes et des phrases. Le structuralisme définit ainsi une méthode combinatoire, qui aboutit à une taxinomie privilégiant l'énoncé réalisé. Il s'agit donc d'une méthode inductivo-déductive : les règles sont induites d'un corpus. Ce recours au corpus explique que l'on ait souvent eu recours à la théorie de l'information et aux statistiques.

Une seconde partie de l'introduction met en évidence les acquis et les limites du structuralisme linguistique. Un consensus s'est fait depuis longtemps pour affirmer que le structuralisme a été à l'origine de la science des langues et des distinctions devenues classiques (synchronie/diachronie ; règles et réalisations individuelles ; rejet du psychologisme et du mentalisme ainsi que de l'ethnocentrisme). Derrière le structuralisme se profile la notion de distributionnalisme ainsi que l'étude statistique des langues et des pathologies du langage. Il reste que le distributionnalisme a des limites qui ont été mises au jour dès les premiers travaux de la grammaire générative : il n'explique pas le phénomène de créativité du langage.

Suit une description rapide des principes de la grammaire générative et transformationnelle élaborée par Chomsky, considérée comme un ensemble de règles, susceptibles d'expliquer un ensemble indéfini de phrases inédites, impliquant de la part du locuteur des jugements de grammaticalité mettant en jeu les notions de compétence et de performance. Sont définies ensuite quelques notions fondamentales comme la compétence et la performance, les transformations, la structure de surface et la structure profonde. Il reste que l'ouvrage n'est pas un exposé complet de la grammaire générative mais en décrit certains aspects en huit chapitres : la phrase minimale (Chap. 1), la dérivation (Chap.2), les nominalisations (Chap. 3), les nominalisation affixales (Chap. 4) et infinitives (Chap. 5), les auxiliaires et les modalités (Chap. 6), la notion de pluriel et de collectif (Chap. 7), les adjectivations (Chap. 8), les diminutifs, augmentatifs et péjoratifs (Chap. 9), les prépositions et la préfixation (Chap. 10). 

générative, mais on n'est pas pour autant en présence d'un «manuel» de grammaire générative. Ce qui est frappant, c'est l'absence totale de bibliographie (à l'exception d'une allusion à N. Ruwet et $\mathrm{M}$. Gross). Une description systématique de la grammaire générative est le fait du volume suivant.

\section{Eléments de linguistique française : syntaxe, par Jean Dubois et Françoise Dubois-Charlier (1970)}

Ce quatrième volume est différent des trois précédents. On note la participation de Françoise Dubois-Charlier. D'autre part, le titre a changé. Il n'est plus question de grammaire structurale mais de linguistique française, avec une référence explicite à la syntaxe. On observera cependant que le titre ne fait aucune référence à la grammaire générative, ce qui est étonnant. Le livre comprend 295 pages et 26 chapitres. A la différence des trois autres, on note la présence d'une bibliographie (sommaire) comprenant N. Chomsky ; N. Ruwet ; R.A. Jacobs et P.S. Rosenbaum, M. Gross et J. Lyons. Ce livre est une présentation de la grammaire générative telle qu'elle était connue en 1970. Le plan est d'une grande clarté. Les trois premiers chapitres sont des présentations de base des concepts de la grammaire générative : la notion de grammaticalité ainsi que la définition de la notion de phrase, de ses constituants et de sa représentation sous forme d'arbres. Le chapitre 3 présente la notion fondamentale de transformations. Les chapitres 4 à 7 sont consacrés au syntagme nominal, à la détermination du nom, aux traits lexicaux ainsi qu'à l'accord en genre et en nombre. Les chapitres 8 à 10 sont dédiés au syntagme verbal, au verbe lui-même (Chap. 9) et à l'auxiliaire (Chap. 10). Le chapitre 11 décrit le syntagme prépositionnel et ses constituants ainsi que certains adverbes, de manière, de lieu et de temps. Le chapitre 12 étudie le syntagme adjectival.

On est étonné de constater que le chapitre 13 est consacré aux constituants de la phrase : on aurait pu penser qu'un tel chapitre ouvrirait le livre. Les chapitres suivants concernent la phrase affirmative (Chap. 15) ainsi que les différentes transformations : le passif (Chap. 16), l'emphase (Chap. 17), la négation (Chap. 18), l'impératif (Chap. 19) et l'interrogation (Chap. 20).

Les derniers chapitres analysent la pronominalisation (Chap.21), les complétives et les circonstancielles (Chap. 22), les relatives (Chap. 23), l'épithète (Chap. 24), les compléments de noms et les possessifs (Chap. 25), les nominalisations (Chap. 26). Le livre est suivi d'un tableau des abréviations utilisées ainsi que d'un index assez détaillé. Une conclusion (rapide) précise que cet ouvrage, étant consacré à la syntaxe, ne traite pas de phonologie ni de sémantique, mais annonce des études ultérieures portant en particulier sur les déterminants et les prépositions.

Avec le recul du temps on peut porter un jugement approprié sur cet ensemble d'ouvrages. Tout d'abord, il faut réaliser l'ampleur de l'entreprise ainsi que son ambition. Il s'agissait de mettre en lumière les modifications théoriques dans un domaine qui était au premier plan dans ces années. On assiste à une démarche alliant l'attrait de la nouveauté et un besoin pédagogique évident. On doit signaler aussi que les informations qui sont communiquées sont le fait de chercheurs américains, dont ces 
ouvrages sont des illustrations. Il reste que ces ouvrages ont été d'une importance particulière pour ceux d'entre nous qui sont entrés en linguistique dans ces années.

\section{BIBLIOGRAPHIE}

Dubois, J. (1965). Grammaire structurale du français : nom et pronom. Paris : Larousse.

Dubois, J. (1967). Grammaire structurale du français : le verbe. Paris : Larousse.

Dubois, J. (1969). Grammaire structurale du français : la phrase et les transformations. Paris : Larousse.

Dubois, J.et Dubois-Charlier, F. (1970). Eléments de linguistique française : syntaxe. Paris : Larousse.

\section{RÉSUMÉS}

L'article rend compte de quatre manuels de Jean Dubois : Grammaire structurale du français : nom et pronom (1965), Grammaire structurale du français : le verbe (1967), Grammaire structurale du français : la phrase et les transformations (1969) et, en collaboration avec F. Dubois-Charlier, Eléments de linguistique française : syntaxe (1970). Ces ouvrages ont fortement marqué toute une génération de chercheurs « entrés en linguistique » entre 1958 et 1970.

This paper presents four textbooks by Jean Dubois three of which are called "structural grammars" of French: Grammaire structurale du français: noms et pronoms (1965), Grammaire structurale du français : le verbe (1967), Grammaire structurale du français : la phrase et les transformations (1969) and Eléments de linguistique française : syntaxe (1970), which was written in collaboration with Françoise Dubois-Charlier. These books strongly influenced a whole generation of researchers who "embraced linguistics" between 1958 and 1970.

\section{INDEX}

Mots-clés : grammaire structurale du français, linguistique françaises des années 1960 et 1970 Keywords : structural grammar of French, French linguistics between 1960 and 1970

\section{AUTEUR \\ GASTON GROSS}

Université Paris 13 